\title{
Head and Neck Schwannomas: A Tertiary Referral Single-Centre Experience
}

\author{
Murat Öztürk' ${ }^{1}$ Seher Șirin ${ }^{1}$, Fidan Rahimli Alekberli ${ }^{1}$, Fatih Mutlu' ${ }^{1}$, Mete İseri $^{1}$ \\ 1 Department of Otorhinolaryngology and Head Neck Surgery, Kocaeli University School of Medicine, Kocaeli, Turkey \\ Murat Öztürk, ORCID: 0000-0002-3340-9975 \\ Seher Sirin, ORCID: 0000-0002-2982-9379 \\ Fidan Rahimli Alekberli, ORCID: 0000-0003-4462-0781 \\ Fatih Mutlu, ORCID: 0000-0003-4831-9582 \\ Mete İseri, ORCID: 0000-0002-3236-4402
}

\begin{abstract}
Objective: The aim is to share our experience of the clinical features and important issues encountered in diagnosing and treating multiple head and neck schwannoma cases seen at our centre, all of which went on to have surgical treatment.
\end{abstract}

Methods: This was a retrospective review of the medical records of cases over an 11-year period (2007-2018) diagnosed with schwannoma by post-surgical histopathology. The demographic characteristics of these cases, along with their clinical characteristics, namely, tumour location, preoperative diagnostic tests undertaken, surgical approach used, and any postoperative complications, including the management of such complications, were reviewed.
Results: A total of 31 patients (18 male, and 13 female) were included in the study. Contrast-enhanced magnetic resonance imaging was the most commonly used pre-operative diagnostic method (77\%). $58 \%$ of the cases were extra- and $42 \%$ intra-cranial. Extracranial schwannomas were noted to arise from several different areas of the head and neck region. The most common neurological deficit post-operatively was facial paralysis.

Conclusion: Since head and neck schwannomas can develop from any area where the nerve sheath is present, they may present with a wide variety of non-specific symptoms. The treatment plan should be made with the anticipated preoperative and postoperative neurological deficit firmly in mind.

Keywords: Schwannoma, head and neck neoplasms, neurilemmoma.
Correspondence: Seher Sirin

Department of Otorhinolaryngology and Head Neck Surgery University of Kocaeli School of

Medicine, 41380, Kocaeli, Turkey E-mail: ugurseher@hotmail.com

Received: 3.10.2019; Accepted: 7.11.2019
Online available at: www.entupdatesjournal.org

* This study was given as an oral presentation at the 40th Turkish National Otorhinolaryngology and

Head Neck Surgery Congress, Antalya, 2018. 


\section{Introduction}

Schwannomas, also known as neurilemmomas, are encapsulated, solitary, benign and slow-growing tumours originating from the sheaths of peripheral, cranial or autonomic nerves. Approximately half of these rare tumours occur in the head and neck region, but they can occur anywhere in the body. ${ }^{[1]}$ They are generally classified according to their anatomical location and the nerve from which they originate. ${ }^{[2]}$ Schwannomas can be extra-, intra- or transcranial. In addition, extracranial schwannomas of the neck are also classified into lateral or medial. While tumours arising from the brachial and cervical plexuses are considered to be lateral, tumours originating from the glossopharyngeal, vagus, accessory, and hypoglossal nerves are considered of medial type. ${ }^{[3]}$

The symptoms and signs of schwannomas vary since they can be found in many different locations within the head and neck region. They typically present with rather non-specific symptoms and therefore are easily overlooked in the differential diagnosis. Malignant transformation is rare but should be suspected if pain or neurological deficits are present. Preoperative diagnostic modalities can provide valuable information and even diagnostic evidence, but a definitive diagnosis requires histopathological examination. ${ }^{[2]}$ However, whilst surgical excision does permit a definitive diagnosis and treatment to occur, there is also the potential for postoperative morbidity. The rarity of tumour occurrence, the uncertain presentation and difficulty in diagnosis, and the potential morbidity associated with surgical treatment mean managing a schwannoma is clinically challenging. Therefore, in this study, we aim to share the expertise gathered in our centre, by outlining the clinical features and the key issues encountered in diagnosis and treatment of head and neck schwannomas that proceeded to surgical treatment.

\section{Materials and Methods}

A retrospective study was undertaken following approval by the Kocaeli University Non-Interventional Ethics Committee (KU/GOKAEK 2018/157). A retrospective review was carried out on cases where schwannoma had been diagnosed histopathologically following surgical excision at the Kocaeli University Otorhinolaryngology and Head \& Neck Surgery Clinic between 2007 and 2018, and who had been followed up regularly in the postoperative period. Cases where non-surgical treatment was employed or follow-up did not occur were excluded from the study. Demographic information on all the cases was recorded, as was the presenting complaint, the clinical features of the schwannoma, the location and nerve of origin, any preoperative diagnostic tests undertaken, the surgical approach followed, postoperative complications and their management, and the duration of follow-up. The gathered data were analysed using the Statistical Package for Social Sciences software version v16.0 (SPSS Inc., Chicago, IL, USA). Categorical variables were expressed as numbers (percentage) and analysed using Pearson's chi-square test. A p value of $<0.05$ was considered statistically significant.

\section{Results}

Thirty-one cases of head and neck schwannoma, who underwent surgical treatment in our tertiary referral clinic between 2007 and 2018 were included in the study.

\section{Demographic Information}

Of the 31 cases, 18 were male and 13 female. The average age was 43.6 years, with an age range of 9 to 65 years. The peak age of tumour occurrence was the $4^{\text {th }}$ decade. There were no significant differences between $m$ ale and female cases in terms of demographic variables. Although male cases predominated in the extracranial group, the result was not statistically significant $(\mathrm{p}=0.439)$. The age and sex distribution of the cases of extracranial and intracranial locations are given in Table 1.

\begin{tabular}{|c|c|c|c|}
\hline $\begin{array}{l}\text { Head and Neck } \\
\text { Schwannomas }\end{array}$ & $\begin{array}{c}\text { Number of patient } \\
\mathrm{n}(\%)\end{array}$ & $\begin{array}{c}\text { Age (years) } \\
\text { Mean (range) }\end{array}$ & $\begin{array}{l}\text { Sex } \\
M / F\end{array}$ \\
\hline Extracranial & $18(58.1)$ & $43.4(9-65)$ & $12 / 6$ \\
\hline Intracranial & $13(41.9)$ & $44(22-61)$ & $6 / 7$ \\
\hline Total & $31(100)$ & $43.6(9-65)$ & $18 / 13$ \\
\hline
\end{tabular}

\section{Location}

Eighteen (58\%) cases were extra- and 13 (42\%) cases intracranial. All the intracranial tumours were vestibular schwannomas. Seven (54\%) tumours were located entirely within the internal acoustic canal, whilst 6 (46\%) tumours extended to the cerebello-pontine angle. Extracranial head and neck schwannomas were for the most part located in the neck region $(n=8,44 \%)$. The other extracranial locations in order of frequency were; middle ear $(n=3)$, tongue $(n=2)$, larynx $(n=2)$, pterygopalatine fossa $(n=1)$, parotid $(\mathrm{n}=1)$ and nasolabial region $(\mathrm{n}=1)$ (Table 2). 


\begin{tabular}{|c|c|c|c|}
\hline Location & Complaint & Surgical Approach & Origin Nerve \\
\hline Extracranial, Neck $\quad(n=8,44 \%)$ & & & \\
\hline Lateral $(n=5,28 \%)$ & $\begin{array}{l}\text { Neck mass }(n=4) \\
\text { Numbness of left hand }(n=1)\end{array}$ & Transcervical $(n=8)$ & $\begin{array}{l}\text { Cervical sympathic chain }(n=3) \\
\text { Brachial plexus }(n=1) \\
\text { Unknown }(n=1)\end{array}$ \\
\hline Medial $(n=3,17 \%)$ & $\begin{array}{l}\text { Neck mass }(n=2) \\
\text { Headache }(n=1)\end{array}$ & & Unknown $(n=3)$ \\
\hline Extracranial, Other $(n=10,56 \%)$ & & & \\
\hline Middle Ear $(n=3,16 \%)$ & Facial paralysis $(n=3)$ & Transmastoid $(\mathrm{n}=3)$ & $\begin{array}{l}\text { Facial Nerve }(n=2) \\
\text { Chorda tympani }(n=1)\end{array}$ \\
\hline Larinx $(n=2,11 \%)$ & Hoarseness $(n=2)$ & Transcervical $(n=2)$ & Unknown $(n=2)$ \\
\hline Tongue $(n=2,11 \%)$ & Tongue Mass $(n=2)$ & Transoral $(n=3)$ & Hypoglossus $(n=2)$ \\
\hline Nasolabial area $\quad(n=1,5 \%)$ & Swelling over upper lip $(n=1)$ & & Unknown $(n=1)$ \\
\hline Parotis $(n=1,5 \%)$ & Swelling below the ear $(n=1)$ & Transcervical $(n=1)$ & Facial Nerve $(n=1)$ \\
\hline Pterygopalatine fossa $(n=1,5 \%)$ & Numbness of the face $(n=1)$ & Transnasal $(n=1)$ & Trigueminal nerve $(n=1)$ \\
\hline $\begin{array}{l}\text { Intracranial } \\
\text { Internal acustic canal }(n=7,54 \%) \\
\text { Cerebellopontine angle }(n=6,46 \%)\end{array}$ & $\begin{array}{l}\text { Dizziness }(n=7,54 \%) \\
\text { Hearing loss }(n=6,46 \%) \\
\text { Tinnitus }(n=6,46 \%)\end{array}$ & $\begin{array}{l}\text { Translabyrinthine }(n=12) \\
\text { Retrosigmoid }(n=1)\end{array}$ & $\begin{array}{l}\text { Superior Vestibular }(n=7) \\
\text { Inferior Vestibular }(n=6)\end{array}$ \\
\hline
\end{tabular}

\section{Clinical Features}

The presenting complaints in the patients with intracranial tumours was dizziness in $7(54 \%)$ and hearing loss in $6(46 \%)$ patients. Amongst the patients with extracranial tumours, the presenting complaints were: neck mass without pain (6 cases, $33 \%$ ); facial paralysis ( 3 cases, $16 \%$ ); hoarseness (2 cases, $11 \%$ ); and tongue mass ( 2 cases, $11 \%$ ). Numbness in the arm, headache, swelling below the ear, numbness on the face and an over-lip mass, were noted less commonly: these symptoms each occurred only once, i.e. in $5 \%$ of cases (Table 2).

\section{Preoperative diagnostic tests}

Contrast-enhanced magnetic resonance imaging (MRI) was the most commonly used diagnostic imaging method $(\mathrm{n}=24,77 \%)$ (Figure 1). All of the 13 cases with intracranial

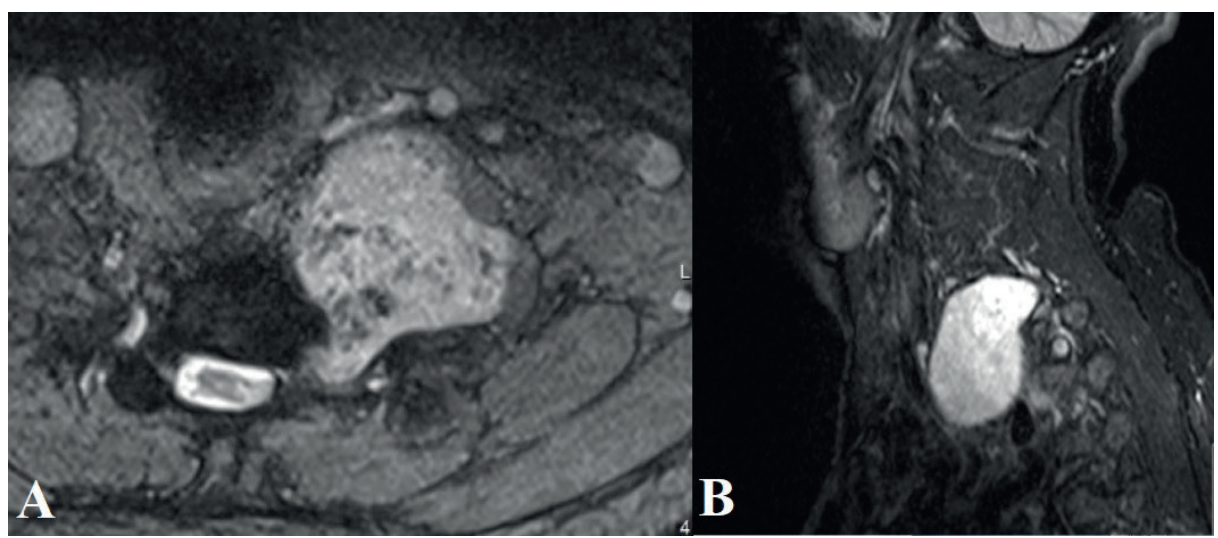

Figure 1. Magnetic resonance imaging of a mass. A. Axial section B. Sagittal section 
head and neck schwannoma were assessed by MRI. In 10 cases, schwannoma was reported as the diagnosis, whilst in a further 3 cases schwannoma was mentioned as a differential diagnosis. Of the 18 cases where the tumour was extracranial, eleven patients were imaged using MRI, three patients were assessed by contrast enhanced computed tomography (CT), and two patients were assessed by Doppler ultrasonography (USG). In two cases, where the tumour was located within the tongue, there was no preoperative imaging. These individuals presented with a smoothly circumscribed polypoid mass visible on the tongue surface. The excised material was reported histopathologically as a schwannoma following a diagnostic total excision performed via a transoral approach under local anaesthesia. Only 3 of the extracranial head and neck schwannomas were directly diagnosed on MRI. One case was reported as a possible pleomorphic adenoma, and in one case the lesion was reported as indistinguishable from a paraganglioma, which was included in the differential diagnosis. For the rest of the cases, 6 patients underwent MRI, three patients CT, and two patients ultrasonography. All (11 cases) were reported as a non-specific mass. Fine needle aspiration cytology (FNAC) was performed in 8 of these cases where the diagnosis was indeterminate. Following FNAC, 2 cases were diagnosed with schwannoma.

\section{Surgical Approach}

In $26(84 \%)$ of cases, the tumour was able to be totally resected, but in $5(16 \%)$ cases where the tumour could not be totally excised, debulking was instead performed.

A trans-labyrinthine approach $(n=12,92 \%)$ was the preferred method in the treatment of the cases of intracranial vestibular schwannoma, with a retrosigmoid approach only used in one case (Table 2). Due to incomplete data, the $3 \mathrm{kHz}$ hearing threshold was not considered when assessing pre- and postoperative hearing. The pure-tone average (PTA) was calculated as the mean of the hearing thresholds at $0.5,1$, and $2 \mathrm{kHz}$. The average PTA of the 12 patients who underwent surgery via a trans-labyrinthine approach was $72 \mathrm{~dB}$ (range:30-118 dB) preoperatively and hearing was unable to be preserved because of the particular surgical technique chosen. The preoperative and postoperative 3rd month PTAs of the single case who underwent surgery via a retrosigmoid approach were $22 \mathrm{~dB}$ and $34 \mathrm{~dB}$, respectively.

For the extracranial head and neck schwannomas, total excision was performed by a transcervical approach in
$11(61 \%)$ patients, transmastoid in $3(17 \%)$, transoral in $3(17 \%)$ and transnasal approach in one $(5 \%)$ patient, the route being chosen according to the location of the lesion (Table 2, Figure 2).

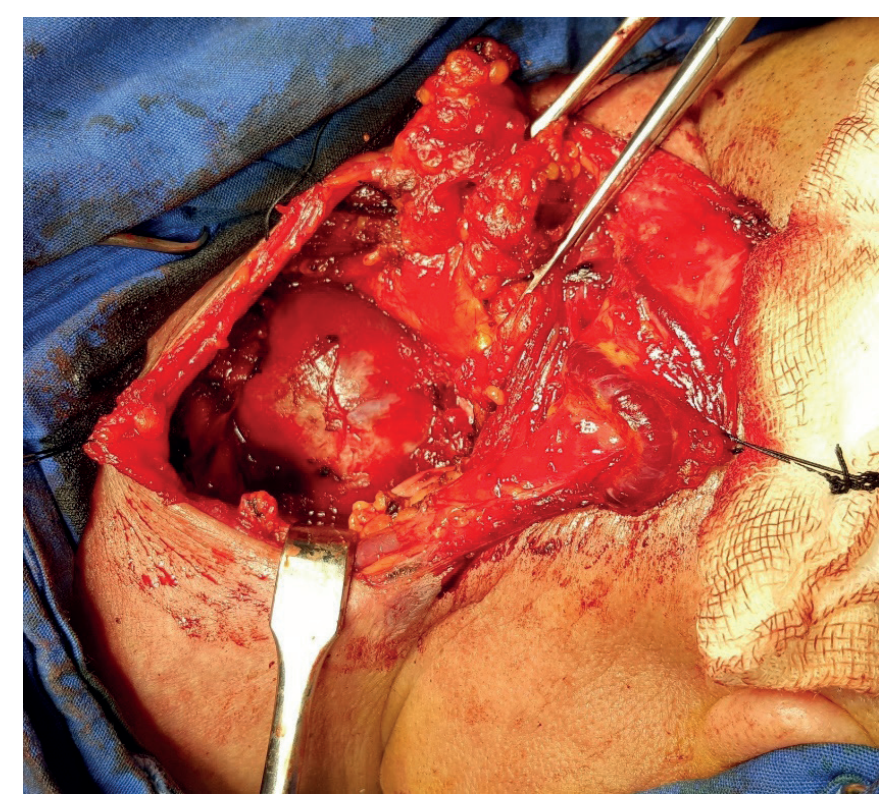

Figure 2. Intraoperative image of cervical tumor.

\section{Nerve origin}

For the intracranial schwannomas, the inferior vestibular nerve was the most common nerve of origin, being found in $6(46 \%)$ such cases. For the extracranial schwannomas, the cervical sympathetic chain in the neck $(n=3,37 \%)$ as well as the facial nerve in other areas $(n=3,30 \%)$ were the most common nerves of origin. In 7 (22\%) patients, the neural origin of the tumour could not be determined with precision (Table 2).

\section{Postoperative complications and management}

Postoperative complications and complaints were reported in $7(23 \%)$ of the vestibular schwannomas treated via a trans-labyrinthine approach. The complications consisted of: dizziness, rhinorrhoea, cerebrospinal fluid (CSF) leak, facial paralysis and Horner syndrome. Facial paralysis was the most common $(n=4,13 \%)$ complication. The management of complications was as follows: pharmacotherapy for vertigo was supplied for one week postoperatively. Rhinorrhoea was noted in one case with facial paralysis, and 
lumbar drainage was performed following neurosurgical advice. Due to ongoing CSF leakage, a revision exploration was performed, and facial nerve decompression with hypoglossal nerve anastomosis were performed. One of the cases with facial paralysis was treated intraoperatively with a gold implant to the upper eyelid. Other patients with facial paralysis received medical treatment and were followed up.

The individual in whom the retrosigmoid approach was used had CSF leakage at the wound site. Following referral to neurosurgery, lumbar drainage was performed and the leakage ceased with conservative treatment. Speech impairment was observed postoperatively in one case, with a CT/MRI evaluation revealing an epidural haematoma. This case was treated by neurosurgery. In another case, where the schwannoma had arisen from the brachial plexus, numbness of the hand developed postoperatively. Physiotherapy to the affected hand resulted in acceptable improvement.

\section{Follow up}

The mean follow-up period for the intracranial cases was 39 months, with a minimum of 5 months and maximum of 67 months follow-up. No recurrence or malignant transformation was detected during the follow-up period. The mean follow-up period of the extracranial cases was 21 months with a minimum one month and maximum 96 months follow-up. No recurrence or malignant transformation was detected during the follow-up period.

Postoperative histopathological appearances were of benign cells with typical spindle cells and hypercellular (Antoni A) and hypocellular (Antoni B) areas, in all cases. Immunohistochemical studies showed S100 positivity in all cases.

\section{Discussion}

Schwann cells are neural crest-derived glial cells that provide myelin isolation to axons of the peripheral nervous system. Schwannomas are benign tumors originating from Schwann cells, first described by Verocay in 1908. ${ }^{[4]} \mathrm{Al}-$ though such tumours may occur at any age, it has been reported that they are most commonly seen between the ages of 20 and 50, with no significant differences in terms of ethnicity or sex. ${ }^{[5-7]}$ In the present study, the majority of patients were between the ages of $20-50$ years $(n=19,61 \%$ ) and the peak occurrence in this sample was in the patients' thirties. Therefore, schwannomas should always be included in the differential diagnosis of head and neck masses in both sexes in both middle and old age.

Since these tumours do not often impair the function of the nerve from which they originate, non-specific symptoms may occur, depending on the location. This results in frequent misdiagnosis of schwannomas. Vagal or glossopharyngeal schwannoma may commonly present with complaints such as dysphonia, dyspnoea, dysphagia, or even cough. Although rare, schwannoma originating from the facial nerve or its surroundings may be the underlying cause of acute peripheral facial paralysis. In our study, three cases presented with facial paralysis, one with facial numbness and another with numbness of the arm. The most common symptom in cases where there was an intracranial vestibular schwannoma was dizziness, and the second most common symptom was hearing loss. In our study, $72 \%$ of cases with an extracranial schwannoma presented with a painless neck mass, which is a very common symptom. Since nerve dysfunction is generally not expected in benign cases, the development of neurological deficits should raise the suspicion of malignancy, which must then be ruled out. This situation also strengthens the surgical indication for these patients. Likewise, a careful radiological screening will be required for local and distant metastasis or other concomitant schwannomas.

Although schwannomas of the head and neck most commonly originate in the cervical sympathetic plexus, they may arise from any of the cranial nerves. Leu and Chang ${ }^{[8]}$ reported on 52 cases of extracranial schwannoma, of which 18 were located in the neck. The origin was reported as the cervical plexus in 9 cases, brachial plexus in 3 cases, cervical sympathetic chain in 2 cases, vagus nerve in 2 cases, hypoglossal nerve in 1 case, and the greater auricular nerve in 1 case $^{[8]}$ Biswas et al ${ }^{[5]}$ reported that in 17 cases out of their series of 31 extracranial schwannomas, the nerve from which the tumour originated could not be identified. The identified origins were reported as the brachial plexus in 5 cases, facial nerve in 3 cases, vagus nerve in 2 cases, hypoglossal nerve in 1 case, cervical sympathetic chain in 1 case, glossopharyngeal nerve in 1 case, and recurrent laryngeal nerve/vagus in 1 case. ${ }^{[5]}$ Hoing et al ${ }^{[9]}$ reported that they were able to find the nerve from which the tumour originated in only 11 of their 20 extracranial schwannoma cases (facial nerve in 4 cases, vagus in 4 cases, and cervical sympathetic chain in 3 cases). In our study, most of the extracranial cases $(n=8,44 \%)$ were located in 
the neck and only three of them had arisen from the cervical sympathetic chain. In addition, we encountered tumours of facial nerve origin in the middle ear as well as tumours of the chorda tympani. The presence of neurological deficits, especially prior to surgery, may be useful in finding the nerve of origin. However, given that most patients are asymptomatic, detection of the nerve of origin of the tumour may depend on high-quality and well-evaluated preoperative imaging methods.

Various imaging methods are in use to evaluate the tumour before intervention. MRI is undoubtedly superior to other imaging modalities in terms of determining the location of the lesion and its relationship with the surrounding soft tissue, as well as for determining the origin of the lesion. Yasumatsu et al ${ }^{[10]}$ reported that the diagnostic sensitivity of MRI was $80 \%$. In our case series, the diagnosis of schwannoma was reported in 13 (54\%) of 24 patients evaluated by MRI. The rate of radiological diagnosis undoubtedly increases with greater experience and interest in this area. We have observed that radiologists, particularly those who have not encountered a schwannoma previously, may overlook schwannoma as a diagnosis, even when typical features are present on imaging. Multidisciplinary evaluation of such cases may improve the differential diagnosis and increase successful recognition of the lesion. FNAC provides benefit in cases where the diagnosis cannot be made by radiological imaging methods alone, but the preoperative diagnostic sensitivity of FNAC alone is inadequate ${ }^{[10]}$ FNAC was used in 8 cases in which the diagnosis was unclear and vascular pathology had been excluded by imaging. Two such cases were reported as schwannoma. FNAC was inadequate to diagnose the disease sufficiently in our case series and the method did not contribute to preoperative diagnosis. Nevertheless, FNAC retains some usefulness as a way to rule out other malignancies.

The management of schwannomas includes both surgical and non-surgical treatment options, such as watchful waiting, complete tumour excision or intracapsular enucleation. Due to the possibility of malignant degeneration and recurrence, it is important to remove the entire mass wherever possible. Therefore, the recommended treatment is usually surgical excision. On the other hand, since tumours arise from the nerve sheath, even with highly delicate and careful surgery, it is very likely that patients who have no neurological deficit prior to surgery may develop neurological deficits postsurgically. Çakır et al ${ }^{[6]}$ reported a failure rate of $57 \%$ in protecting the nerve of origin in their extracranial head and neck schwannoma cases. Therefore, the surgical decision should be informed by an evaluation of the nerve of origin and its likely impact on the patient's quality of life if a postoperative deficit occurs. While nerve dysfunction is more acceptable in cases where the tumour arises from a small sensory nerve, deficits in major nerves and the cranial nerves will be more apparent. In our study, $19 \%$ of the cases reviewed had significant neural deficits after surgery. Facial paralysis developed in 4 such patients, although the deficit improved partially in the late postoperative period. In one patient with a brachial plexus-derived tumour, postoperative numbness in the hand improved acceptably with physiotherapy. Giving detailed information before surgery and planning rehabilitation strategy in the postoperative period facilitates the management of the complications and increases success. For all these reasons, the surgical decision should be made considering the balance between risks and benefits, i.e. by comparing the severity of preoperative symptomatology and the anticipated postoperative neurological deficit. In current treatment management algorithms ${ }^{[11]}$, asymptomatic benign lesions have been reported to be good candidates for observation as long as they remain stable Our general approach in clinical practice is to observe asymptomatic extracranial masses, especially those located medially, in patients who can be relied on to attend follow-up.

Even some cases treated surgically required careful postoperative follow-up, with annual MRI. It is very important to inform the patient in detail about what symptoms and signs may develop due either to the disease or to treatment, and to advise the individual to attend hospital without waiting for a follow-up appointment if any additional symptoms occur. The follow-up period should also include the evaluation, follow-up and rehabilitation of treatment-related deficits and complications. The effects of neurological deficits may change or worsen with age, plus further disease is a possibility in young individuals with otherwise full life expectancy. Long-term follow-up should be planned around these issues.

\section{Conclusion}

Head and neck schwannomas are rare tumours which may present with multiple various and non-specific symptoms, since they can develop from any area where nerves are present. The treatment decision should be made on the basis of the potential neurological deficits that exist preoperatively, and those anticipated postoperatively. Therefore, accurate 
preoperative diagnosis with secure identification of the nerve from which the tumour originates allows patients to be informed about possible nerve damage and make an informed decision about accepting surgery or follow-up. In preoperative diagnostic imaging, MRI is superior to other imaging modalities in determining the relationship of the lesion with the surrounding tissue, its location and especially in identifying the nerve of origin. FNAC may provide additional benefit for the differential diagnosis and may allow the recognition of other pathologies. If surgical excision is planned, total excision of the tumour should be performed and a multidisciplinary team should then work together to address potential postoperative morbidity.

Acknowledgement: The authors would like to thank Canan Baydemir for statistical support.

\section{References}

1. Das Gupta TK, Brasfield RD, Strong EW, Hajdu SI. Benign solitary schwannomas (neurilemomas) Cancer 1969;24:355-66.

2. Zhang H, Cai C, Wang S, Liu H, Ye Y, Chen X. Extracranial head and neck schwannomas: a clinical analysis of 33 patients. Laryngoscope 2007;117:278-81.

3. Colreavy MP, Lacy PD, Hughes J, et al. Head and neck schwannomas--a 10 year review. J Laryngol Otol 2000;114:119-24.

4. Verocay J. Multiple Geschwulste als Systemerkrankung am nervosen Apparate. Dittrich P ed. In: Chiari H, editor. Festchrift Herrn Hofrat. Wien and Leipzig: W. Braumuller; 1908. p. 3421-52. [Book chapter in German]

5. Biswas D, Marnane CN, Mal R, Baldwin D. Extracranial head and neck schwannomas-a 10-year review. Auris Nasus Larynx 2007;34:353-9.

6. Çakır A, Erdağ TK, Ecevit MC, Güneri EA, Sütay S, İkiz AÖ. Extracranial head and neck schwannomas: A series of 14 patients. Kulak Burun Bogaz Ihtis Derg 2017;27:257-63.
Ethics Committee Approval: Ethical approval was obtained from the Institutional Review Board of the Kocaeli University, School of Medicine (KU/GOKAEK 2018/157).

Informed Consent: Informed consent was obtained from all individual participants included in the study.

Author Contributions: Designing the study - M.Ö., S.Ş., F.R.A.; Collecting the data - M.Ö., S.Ş., F.R.A.; Analyzing the data M.Ö., S.Ş., F.R.A., F.M., M.İ.; Writing the manu-script - M.Ö., S.Ş., F.R.A., F.M., M.İ.; Confirming the accura-cy of the data and the analyses - M.Ö., S.Ş., F.R.A., F.M., M.İ.

Conflict of Interest: The authors have no conflicts of interest to declare.

Financial Disclosure: The authors declare that no financial support was received for this paper.
7. Altuntaş EE, Bora A, Cerrah YSS, Müderris S, Elagöz Ş. Evaluation of the head and neck schwannoma's in our clinic. [Article in Turkish] Cumhuriyet Tip Derg 2012;34:289-94.

8. Leu YS, Chang KC. Extracranial head and neck schwannomas: a review of 8 years experience. Acta Otolaryngol 2002;122:435-7.

9. Hoeing B, Stuck BA, Kansy B, et al. Extracranial schwannomas of the Head and Neck: clinical approach based on 20 cases. [Article in German] Laryngo Rhino Otol 2017;96:607-14.

10. Yasumatsu R, Nakashima T, Miyazaki R, Segawa Y, Komune S. Diagnosis and management of extracranial head and neck schwannomas: a review of 27 cases. Int J Otolaryngol 2013;2013:973045.

11. Yafit D, Horowitz G, Vital I, Locketz G, Fliss DM. An algorithm for treating extracranial head and neck schwannomas. Eur Arch Otorhinolaryngol 2015;272:2035-8.

This is an open access article distributed under the terms of the Creative Commons Attribution-NonCommercial-NoDerivs 3.0 Unported (CC BY- NC-ND3.0) Licence (http://creativecommons.org/licenses/by-nc-nd/3.0/) which permits unrestricted noncommercial use, distribution, and reproduction in any medium, provided the original work is properly cited.

Please cite this article as: Öztürk M, Şirin S, Rahimli Alekberli F, Mutlu F, İșeri M. Head and Neck Schwannomas: A Tertiary Referral Single-Centre Experience. ENT Updates 2019;9(3): 206-212. 